\title{
Substructure in the Frontier Fields from weak lensing flexion
}

\author{
Markus Rexroth \\ Laboratoire d'Astrophysique, Ecole Polytechnique Fédérale de Lausanne (EPFL), Observatoire \\ de Sauverny, CH-1290 Versoix, Switzerland
}

\begin{abstract}
Flexion is the second order weak gravitational lensing effect responsible for the arclike appearance of sources. It is highly sensitive to dark matter substructure and can greatly increase the resolution of mass maps, but it is very hard to measure. We present an automated flexion measurement pipeline for Hubble Space Telescope data and a preliminary application to the Frontier Fields cluster MACSJ0416.1-2403.
\end{abstract}

Keywords. Gravitational lensing, dark matter, clusters: individual (MACSJ0416.1-2403)

\section{Introduction}

In weak lensing, the unlensed 2-dimensional coordinates $\beta_{i}$ and the lensed, observed coordinates $\theta_{i}$ are to first order related by $\beta_{i}=A_{i j} \theta_{j}$, where $A_{i j}=\partial \beta_{i} / \partial \theta_{j}$ is expressed in terms of the convergence $\kappa$ and the shear $\gamma$. This approximation only holds if $\kappa$ and $\gamma$ are constant over a lensed image. Otherwise we have to expand the relation by including flexion: $\beta_{i}=A_{i j} \theta_{j}+\frac{1}{2} D_{i j k} \theta_{j} \theta_{k}$, where $D_{i j k}=\mathcal{F}_{i j k}+\mathcal{G}_{i j k}$ is the sum of the F-Flexion (spin-1) and the G-Flexion (spin-3) terms (Bacon et al. (2006), Goldberg \& Natarajan (2002), Irwin \& Shmakova (2006)). The F-Flexion shifts the centroid of a lensed source and the G-Flexion makes it triangular. The flexions are responsible for the arclets close to strong lenses. We cannot measure flexion itself in real data, but only reduced flexion, $F=\mathcal{F} /(1-\kappa)$ and $G=\mathcal{G} /(1-\kappa)$ (Schneider \& Er (2008)). Adding flexion to weak lensing has great advantages. Typically $\kappa$ and $\gamma$ decline as $r^{-1}$, while flexion drops off as $r^{-2}$. Thus it is much more sensitive to small scale structure and weak lensing mass maps will have a much higher resolution (Leonard et al. (2009), Bacon et al. (2010)). Magnification maps will be more accurate. Furthermore, flexion allows us to measure signals close to the strong lensing region and thus bridges the gap between strong and weak lensing. It was demonstrated that measurements in simulations or in the strong lensing cluster Abell 1689 are in principle possible (e.g., Leonard et al. (2007), Okura et al. (2008), Rowe et al. (2013)). However, measurements in real data have proved to be difficult and to this day, no public measurement pipeline exists. Therefore we have developed an automated, efficient flexion pipeline for Hubble Space Telescope (HST) data.

\section{An automated flexion measurement code for $H S T$ data}

The fully automated pipeline uses the HOLICs flexion measurement technique (Okura et al. (2007), Okura et al. (2008), Goldberg \& Leonard (2007)). It extends the KSB shear extraction technique (Kaiser et al. 1995) by including higher order image moments. In addition, our code discards overlapping sources and subtracts background noise. Flexion measurements depend on several variables, e.g. source size, signal-to-noise, and morphology (Viola et al. (2012), Rowe et al. (2013)). As a result, the measurement error is hard to estimate and several potential biases can arise. Therefore we have created simulated 

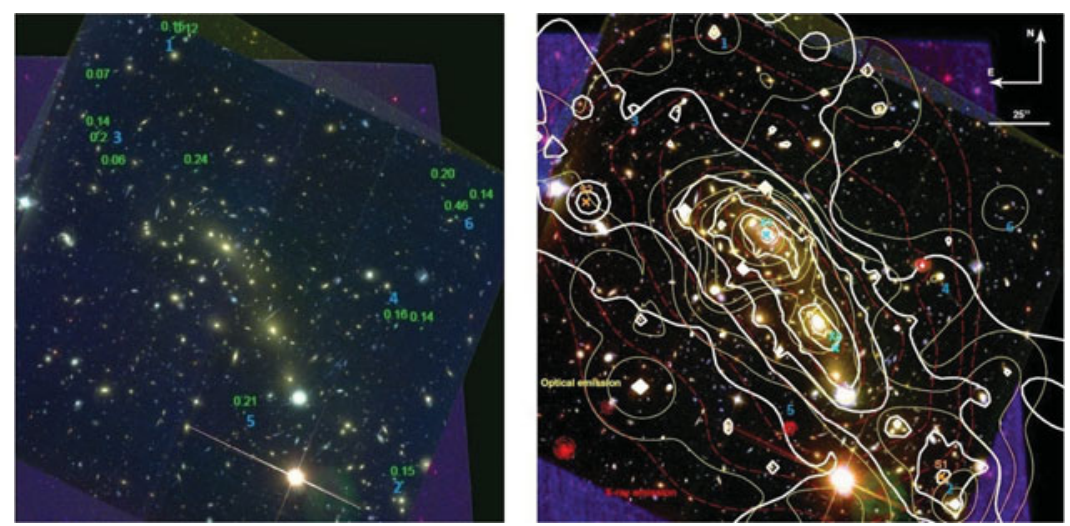

Figure 1. Preliminary F-Flexion magnitudes in the cluster MACSJ0416.1-2403 (green, left) confirm 4 substructures of the Jauzac et al. (2015) mass model (blue, 1 to 4 ) and find 2 new candidate dark matter clumps (blue, 5 and 6 ). White contours show the mass model, yellow lines indicate the light distribution and red contours outline the X-ray surface brightness.

images of galaxies with a wide range of different properties and flexions. We use this simulation to calibrate the pipeline, $F_{\text {true }}=m \cdot F_{\text {meas }}+c$, and analogously for G-Flexion. In addition, our code will provide a measurement error estimate.

\section{Preliminary results: Application to the cluster MACSJ0416.1-2403}

We applied our flexion pipeline to the Frontier Fields cluster MACSJ0416.1-2403. As the calibration which accounts for bias effects was not yet applied, we used only the 14 largest, most reliable sources. The measurements including the calibration of a larger sample of background galaxies will be presented in our forthcoming paper (Rexroth et al. (2015) in prep.). The F-Flexion confirms several substructures predicted by the high precision mass model presented in Jauzac et al. (2015), see Figure 1. We also find 2 new candidate dark matter clumps which the mass model could not constrain. The GFlexion has to our knowledge never been measured in real data. We measure a G-Flexion signal that is compatible with the F-Flexion results, but has higher measurement errors. Our results show that already a small flexion sample can greatly improve mass maps.

\section{References}

Bacon, D., Amara, A., \& Read, J. 2010, MNRAS, 409, 389

Bacon, D., Goldberg, D., Rowe, B., \& Taylor, A. 2006, MNRAS, 365, 414

Goldberg, D. \& Leonard, A. 2007, ApJ, 660, 1003

Goldberg, D. \& Natarajan, P. 2002, ApJ, 564, 65

Irwin, J. \& Shmakova, M. 2006, ApJ, 645, 17

Jauzac, M., Jullo, E., Eckert, D., et al. 2015, MNRAS, 446, 4132

Kaiser, N., Squires, G., \& Broadhurst, T. 1995, ApJ, 449, 460

Leonard, A., Goldberg, D., Haaga, J., \& Massey, R. 2007, ApJ, 666, 51

Leonard, A., King, L., \& Wilkins, S. 2009, MNRAS, 395, 1438

Okura, Y., Umetsu, K., \& Futamase, T. 2007, ApJ, 660, 995

Okura, Y., Umetsu, K., \& Futamase, T. 2008, ApJ, 680, 1

Rowe, B., Bacon, D., Massey, R., et al. 2013, MNRAS, 435, 822

Schneider, P. \& Er, X. 2008, A\&A, 485, 363

Viola, M., Melchior, P., \& Bartelmann, M. 2012, MNRAS, 419, 2215 